\title{
Pronto: A Non-monotonic Probabilistic Description Logic Reasoner
}

\author{
Pavel Klinov \\ The University of Manchester, Manchester, M13 9PL, UK \\ pklinov@cs.man.ac.uk
}

\begin{abstract}
The demonstration presents Pronto - a prototype of a nonmonotonic probabilistic reasoner for very expressive Description Logics. Pronto is built on top of the OWL DL reasoner Pellet, and is capable of performing default probabilistic reasoning in the Semantic Web. It can handle uncertainty in terminological and assertional DL axioms. The demonstration covers Pronto's features and capabilities as well as current challenges and limitations. It describes how an involved realistic problem of breast cancer risk assessment can be formalized in terms of probabilistic reasoning in Pronto. As an important outcome, it is anticipated that attendees should learn and better understand the potential of ontology based approaches to modeling problems involving reasoning under uncertainty.
\end{abstract}

\section{Introduction}

One of the limitations of current Description Logic (DL) reasoners is the inability to handle uncertain knowledge. It is a serious obstacle to the expansion of the Semantic Web because many domains of human interest contain knowledge that cannot be represented with absolute certainty. One example of an uncertain domain is medicine, in particular, disease diagnosing. Symptoms, causes and consequences of many diseases are uncertain which complicates conceptualization of such domains in formal ontologies and thus restricts machine understanding.

This demonstration presents Pronto - a probabilistic DL reasoner prototype [1]. Pronto is an attempt to provide reasoning services for $\mathrm{P}-\mathcal{S H \mathcal { I N }}(\mathrm{D})$ - a very expressive formalism that is a probabilistic generalization of OWL with the exception of nominals [2]. In addition to presenting the Pronto's features and capabilities, the demo displays how Pronto can aid in the probabilistic modeling of a realistic medical problem by providing representation and reasoning services for constructing and using a probabilistic ontology for breast cancer risk assessment.

\section{Research Background}

Pronto provides means for the representation of uncertain ontological statements and offers a collection of reasoning services. It uses the syntax of Lukasiewicz's conditional constraints to express uncertain OWL axioms 2 and Lehmann's lexicographic entailment to perform default probabilistic reasoning [3] 22. 


\subsection{Representation of Uncertain Knowledge}

Pronto represents uncertain ontological knowledge using $\mathrm{P}-\mathcal{S} \mathcal{H} \mathcal{I} \mathcal{N}(\mathrm{D})$ formalism 2 that is a probabilistic generalization of the very expressive DLs $\mathcal{S H \mathcal { H }}(\mathrm{D})$. It is heavily based on the earlier developed approach to default probabilistic reasoning with conditional constraints [4. In the context of $\mathrm{P}-\mathcal{S H \mathcal { I N }}(\mathrm{D})$, conditional constraints are expressions of the form $(D \mid C)[l, u]$ where $C$ and $D$ are arbitrary $\mathcal{S H \mathcal { N } N}(\mathrm{D})$ concepts and $[l, u]$ is a closed interval within $[0,1]$.

Using conditional constraints Pronto is capable of representing both, generic and individual uncertainty. For example, a generic constraint $($ Fly $\mid$ Bird $)[0.9,0.95]$ where Fly and Bird are DL concepts, can express the uncertainty that randomly picked bird can fly. Similarly, a constraint (Penguin $\mid \top)[0.7,0.8]$ for individual Tweety captures the uncertainty in Tweety being an instance of Penguin.

The important feature of $\mathrm{P}-\mathcal{S H \mathcal { H }} \mathcal{N}(\mathrm{D})$ supported by Pronto is the ability to capture default terminological knowledge. That is, TBox constraints are default in the sense that they represent statements that are generally true but might fail for some specific individuals. They can also be overridden by more specific statements. The demo will exemplify this possibility.

\subsection{Default Probabilistic Reasoning}

The main reasoning task in $\mathrm{P}-\mathcal{S H \mathcal { H }} \mathcal{N}(\mathrm{D})$ is entailing new conditional constraints, both, terminological and assertional, from probabilistic knowledge bases. Given that standard notion of logical entailment is too weak [4, it has been proposed to use Lehmann's lexicographic entailment that obeys desirable non-monotonic properties [3. In particular, it allows to resolve conflicts between conditional constraints by preferring some constraints to others 4 .

Computing probabilistic entailments involves other reasoning procedures, such as probabilistic satisfiability and probabilistic consistency. Satisfiability is defined in a traditional way, i.e., as a problem of determining whether given probabilistic knowledge base has a model. Consistency has no analogue in monotonic DL reasoning. Knowledge base is consistent iff given a probabilistic entailment relation, it is possible to resolve all conflicts during reasoning.

All aforementioned reasoning procedures are implemented in Pronto.

\section{Technological Basis}

One of the principal requirements for Pronto was that the uncertainty could be gradually introduced into existing OWL ontologies and that the existing OWL reasoning services should be retained. To meet that requirement, Pronto was designed on top of the OWL reasoner Pellet [5] that performs reasoning with the classical part of ontologies and provides routines for higher level probabilistic reasoning procedures, e.g., lexicographic entailment.

Pronto also employs methods for solving linear optimization and related subproblems. Following Lukasiewicz's approaches to probabilistic default reasoning 
and probabilistic logic programming, Pronto reduces the probabilistic satisfiability (PSAT) to the problem of solvability of a corresponding linear system. In addition, lexicographic entailment is reduced to a number of logical entailments each of which is computed by performing linear optimization. Pronto makes use of an LP solver to perform these tasks 1 .

Finally, Pronto uses OWL 1.1 axiom annotations to associate probability intervals with uncertain OWL axioms.

\section{Demonstration}

It has been chosen to use Life Sciences domain, in particular, medical informatics to demonstrate Pronto's capabilities. Medical informatics has a successful history of using ontologies for modeling domain knowledge [6]. At the same time, medical domain knowledge is often uncertain especially when it is required to diagnose diseases or estimate risks of developing them in the future.

One relevant medical problem is assessing women risk of developing breast cancer. Given that neither all the relevant risk factors are known nor their impact is sufficiently investigated, the assessment cannot be done with absolute certainty. There have been proposed few statistical approaches to probabilistically estimate the breast cancer risk, for example, Gail model used by the National Cancer Institute (NCI) risk calculator 2 [7.

The demo aims to show how Pronto might aid in approaching the same problem by incorporating the statistical knowledge into a cancer ontology. It will first present how the problem can be modeled using a probabilistic ontology, and second, how risk assessment can be performed on the basis of probabilistic default reasoning. Extended version of web based ontology browser OWLSight 3 will serve as the interface to Pronto.

\subsection{Probabilistic Model Demonstration}

The model of breast cancer risk assessment consists of two major parts - classical OWL ontology and the probabilistic part that represents domain uncertainty. The classical part of the ontology models two types of risk of developing breast cancer. First is absolute risk, i.e., the risk that can be measured without the reference to other categories of women. Statements like" an average woman has up to 13.2\% of developing breast cancer in her lifetime" are the examples of the absolute risk. Second, the ontology models relative breast cancer risk, i.e., the risk comparably to an average woman. Statements like "having BRCA1 gene mutation increases the risk of developing breast cancer by a factor of four" are the examples of the relative risk.

The ontology defines risk factors that are relevant to breast cancer. It makes the distinction between the factors that should be known to a woman, e.g., age,

\footnotetext{
${ }^{1}$ Operations Research library, available at: http://opsresearch.com/OR-Objects/

${ }^{2}$ http://www.cancer.gov/bcrisktool

${ }^{3}$ Standard version is available at: http://pellet.owldl.com/ontology-browser/
} 
family cancer history, breastfeeding, and those that can only be inferred on the basis of other factors or by the examination, e.g., BRCA gene mutation, breast and bone densities, etc.

Following the assumption that the subjective probabilities representing risk factors for a certain individual can be combined with objective probabilities representing the statistical knowledge, the probabilistic part combines a set of uncertain ABox and TBox axioms 4. ABox axioms define risk factors that are relevant to a particular individual. TBox axioms model generic probabilistic relationships between the risk factors and classes of women.

It will be shown how to express various dependencies between risk factors. One possibility is to represent how the presence of one risk factor allows to guess on the presence of others. This is the principal way to use inferred risk factors, i.e., those unknown to a woman. For example, it is known that Ashkenazi Jews are more likely to develop BRCA gene mutation.

It will be demonstrated how to capture the impact of combining risk factors, i.e., if they are known to strengthen or weaken each other. Classical part of the ontology provides classes that are combinations of multiple risk factors. The model can define the risk for women having multiple risk factors to be higher (or lower) than if they had just one of the factors. This is possible using the overriding feature of the default probabilistic reasoning.

Finally, the ontology contains a number of ABox axioms that represent risk factors for specific individuals. The motivation is that while the generic probabilistic model that provides all the necessary statistics can be developed and maintained by a central cancer research institute, individual women can supply the knowledge about the risk factors that are known to them, e.g., age. It will also be shown how to express uncertainty in having some particular risk factor.

\subsection{Probabilistic Reasoning Demonstration}

The modeling described above is necessary to reduce the problem of assessing breast cancer risks to the standard lexicographic entailment implemented in Pronto. Risk assessment for a particular woman corresponds to the entailment of an ABox constraint. For example, (WomanWithBRCInLongTerm $\mid T)[0.6,0.8]$ implies that some woman's risk of developing cancer in life time is $60 \%-80 \%$. The reasoning will be demonstrated on a number of test probabilistic individuals.

It will also be presented how Pronto justifies the results of the risk assessment by generating the explanations for the entailments. In particular, it can retrieve exactly those risk factors and generic statistical axioms that caused the inference for a particular woman and filter our all the irrelevant risk factors. In addition to being useful for end users, this capability can aid the model developers in testing the accuracy and adequacy of their model.

Finally, the demo will reveal some pitfalls of default probabilistic reasoning by presenting seemingly unobvious, yet sound entailments. This will provide a better insight into the nature of probabilistic reasoning and also demonstrate the need of explanations.

\footnotetext{
${ }^{4}$ Available at: http://www2.cs.man.ac.uk/ ${ }^{k \text { klinovp/pronto/brc/cancer_cc.owl }}$
} 


\section{Discussion}

Although the demo does not pretend to cover all the aspects of default probabilistic reasoning in the Semantic Web, it is expected to help the attendees learn the following important things:

- Features and capabilities of Pronto. The demo will present Pronto's fundamental features, e.g., reasoning services and representational capabilities.

- How Pronto can help in modeling real life problems that involve uncertain knowledge. It is important to learn that the limits of applicability of ontologies will be pushed forward once they become capable of representing uncertain knowledge. The breast cancer risk assessment is one of the problems that can be given a probabilistic ontological model and then approached using a Semantic Web reasoner.

- How current OWL ontologies can be reused in probabilistic models.

- Current challenges and limitations of probabilistic reasoning as well as future research directions.

Acknowledgment. Pronto was developed when the first author was an intern at Clark \& Parsia, LLC (www.clarkparsia.com) during the summer 2007. Pronto is a property of Clark \& Parsia and is available under the AGPL license at: http://pellet.owldl.com/pronto. The authors are thankful to Bijan Parsia, Evren Sirin, Michael Smith and Kendall Clark for numerous useful suggestions and other support regarding the nature of this work.

\section{References}

1. Klinov, P., Parsia, B., Mazlack, M.J.: Pronto - a non-monotonic probabilistic description logic reasoner. In: The European Semantic Web Conference (submitted, 2008)

2. Lukasiewicz, T.: Probabilistic description logics for the semantic web. Technical Report Nr. 1843-06-05, Institut fur Informationssysteme, Technische Universitat Wien (2007)

3. Lehmann, D.: Another perspective on default reasoning. Annals of Mathematics and Artificial Intelligence 15(1), 61-82 (1995)

4. Lukasiewicz, T.: Probabilistic default reasoning with conditional constraints. Annals of Mathematics and Artificial Intelligence 34(1-3), 35-88 (2002)

5. Sirin, S., Parsia, B., Grau, B.C., Kalyanpur, A., Katz, Y.: Pellet: A practical OWLDL reasoner. Technical Report CS 4766, University of Maryland, College Park, MD (2005)

6. Golbeck, J., Fragoso, G., Hartel, F.W., Hendler, J.A., Oberthaler, J., Parsia, B.: The national cancer institute's thesaurus and ontology. Journal of Web Semantics 1(1), 75-80 (2003)

7. Gail, M.H., Brinton, L.A., Byar, D.P., Corle, D.K., Green, S.B., Shairer, C., Mulvihill, J.J.: Projecting individualized probabilities of developing breast cancer for white females who are being examined annually. Journal of the National Cancer Institute 81(25), 1879-1886 (1989) 